\title{
On the structure of quantum $\mathrm{L}_{\infty}$ algebras
}

\section{Ralph Blumenhagen, Michael Fuchs and Matthias Traube}

Max-Planck-Institut für Physik (Werner-Heisenberg-Institut), Föhringer Ring 6, 80805 München, Germany

E-mail: blumenha@mpp.mpg.de, mfuchs@mpp.mpg.de, mtraube@mpp.mpg.de

ABSTRACT: It is believed that any classical gauge symmetry gives rise to an $\mathrm{L}_{\infty}$ algebra. Based on the recently realized relation between classical $\mathcal{W}$ algebras and $\mathrm{L}_{\infty}$ algebras, we analyze how this generalizes to the quantum case. Guided by the existence of quantum $\mathcal{W}$ algebras, we provide a physically well motivated definition of quantum $\mathrm{L}_{\infty}$ algebras describing the consistency of global symmetries in quantum field theories. In this case we are restricted to only two non-trivial graded vector spaces $X_{0}$ and $X_{-1}$ containing the symmetry variations and the symmetry generators. This quantum $\mathrm{L}_{\infty}$ algebra structure is explicitly exemplified for the quantum $\mathcal{W}_{3}$ algebra. The natural quantum product between fields is the normal ordered one so that, due to contractions between quantum fields, the higher $\mathrm{L}_{\infty}$ relations receive off-diagonal quantum corrections. Curiously, these are not present in the loop $\mathrm{L}_{\infty}$ algebra of closed string field theory.

KEywords: Conformal and W Symmetry, Conformal Field Models in String Theory

ARXIV EPRINT: 1706.09034 


\section{Contents}

1 Introduction 1

2 The $\mathrm{L}_{\infty}$ gauge algebra of a classical symmetry 2

3 Quantum $\mathrm{L}_{\infty}$ gauge algebras $\quad 5$

3.1 The quantum $\mathrm{L}_{\infty}$ algebra of a quantum symmetry 5

3.2 Comparison to the $\mathrm{L}_{\infty}$ algebra of CSFT 9

4 The quantum $\mathcal{W}_{3}-\mathrm{L}_{\infty}$ algebra $\quad 11$

$\begin{array}{lll}4.1 \mathcal{W} \text { algebras } & 11\end{array}$

$\begin{array}{lll}4.2 & L_{n} \text { products with one symmetry parameter } & 12\end{array}$

$\begin{array}{lll}4.3 & L_{n} \text { products with two symmetry parameters } & 13\end{array}$

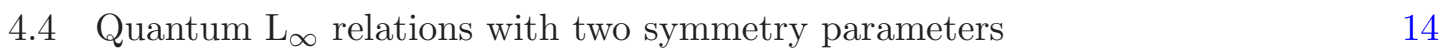

$\begin{array}{lll}4.5 & \mathrm{~L}_{\infty} \text { relations with three symmetry parameters } & 18\end{array}$

$\begin{array}{llr}5 & \text { Summary and conclusions } & 19\end{array}$

\section{Introduction}

Derived from closed string field theory [1], the structure of $\mathrm{L}_{\infty}$ algebras were suggested to underly all classical perturbative gauge symmetries and their dynamics. For the first time, they actually appeared in the context of higher spin gauge theories [2] and were also discussed in the mathematics literature (see e.g. [3-6]). Motivated by the study of field theory truncations of string field theory [7], the authors of [8] argued that the symmetry and the action of any consistent perturbative gauge symmetry is controlled by an $\mathrm{L}_{\infty}$ algebra. For Chern-Simons and Yang-Mills gauge theories as well as for double field theory the symmetries and equations of motion could be expressed in terms of an $\mathrm{L}_{\infty}$ structure.

Based on the higher spin $\mathrm{AdS}_{3}-\mathrm{CFT}_{2}$ duality, a large set of explicit non-trivial $\mathrm{L}_{\infty}$ algebras were identified recently [9] by showing that the well understood class of classical $\mathcal{W}$ algebras can also be rewritten in terms of higher products satisfying the relations of $\mathrm{L}_{\infty}$ algebras. Recall that $\mathcal{W}$ algebras appear as extended chiral symmetry algebras of two-dimensional conformal field theories (CFTs)(see [10] for a review) and are actually not describing gauge symmetries but infinitely many global symmetries. These examples are special in the sense that only two graded vector spaces were non-trivial, $X_{0}$ contains the symmetry parameters and $X_{-1}$ the generators of the $\mathcal{W}$ algebra. The special feature of $\mathcal{W}$ algebras, namely that the Poisson bracket between the generators closes only non-linearly, implied non-trivial higher products, corresponding e.g. to field dependent symmetry parameters. 
In [9] this correspondence was restricted to the classical case, for which the product of fields is just the point-wise product of holomorphic functions. However, from CFT it is well known that these classical $\mathcal{W}$ algebras appear as the classical $\hbar \rightarrow 0$ limit of quantum $\mathcal{W}$ algebras. Here one is dealing with chiral quantum fields, whose product involves a normal ordering prescription. In addition, the field content of the algebra itself and their structure constants receive $\hbar$ corrections.

It is an interesting question, how the $\mathrm{L}_{\infty}$ structure generalizes to the quantum case. In the context of string field theory, this was already analyzed in [1] and further elucidated in the mathematical context in [11]. In this paper we generalize the analysis of [9] to quantum $\mathcal{W}$-algebras. We will see that the higher products now involve the normal ordered product as the fundamental one, and that they also receive $\hbar$ corrections. In addition also the quadratic relations among the higher products receive quantum corrections, induced by non-trivial contractions following from the application of Wick's theorem. Since we are dealing with an interacting (non-free) CFT, these contractions are given by the singular part of the operator product expansion (OPE) and, as will be shown, imply off-diagonal terms among the naive classical $\mathrm{L}_{\infty}$ relations. Guided by quantum $\mathcal{W}$ algebras we are thus led to a well motivated definition of quantum $\mathrm{L}_{\infty}$ algebras that control the symmetries of a quantum theory. Similar as in the case of classical symmetries the quantum $\mathrm{L}_{\infty}$ algebras we look at are restricted to a graded vector space $X=X_{0} \oplus X_{-1}$ and are constructed such that they become the classical $\mathrm{L}_{\infty}$ algebra of the classical symmetry in the $\hbar \rightarrow 0$ limit.

The paper is organized as follows: in section 2 we recall the definition of a classical $\mathrm{L}_{\infty}$ algebra and its connection to the gauge algebra of classical gauge field theories. In section 3 , after identifying the possible origin of quantum corrections, we first define quantum $\mathrm{L}_{\infty}$ algebras. Then we will compare it to loop $\mathrm{L}_{\infty}$ algebras, the quantum corrected $\mathrm{L}_{\infty}$ algebras arising for closed string field theory (CSFT) $[1,11]$. In section 4 we will show in detail that the quantum $\mathcal{W}_{3}$ algebra is organized in terms of a quantum $\mathrm{L}_{\infty}$ algebra.

\section{The $\mathrm{L}_{\infty}$ gauge algebra of a classical symmetry}

In this section we review how a perturbative classical gauge algebra is actually controlled by an $\mathrm{L}_{\infty}$ algebra. $\mathrm{L}_{\infty}$ algebras are generalized Lie algebras where one has not only a two-product, the commutator, but more general multilinear $n$-products with $n$ inputs

$$
\begin{aligned}
\ell_{n}: \quad X^{\otimes n} & \rightarrow X \\
x_{1}, \ldots, x_{n} & \mapsto \ell_{n}\left(x_{1}, \ldots, x_{n}\right),
\end{aligned}
$$

defined on a graded vector space $X=\bigoplus_{n} X_{n}$, where $n$ denotes the grading. The products are graded symmetric

$$
\ell_{n}\left(\ldots, x_{1}, x_{2}, \ldots\right)=(-1)^{1+\operatorname{deg}\left(x_{1}\right) \operatorname{deg}\left(x_{2}\right)} \ell_{2}\left(\ldots, x_{2}, x_{1}, \ldots\right),
$$

with

$$
\operatorname{deg}\left(\ell_{n}\left(x_{1}, \ldots, x_{n}\right)\right)=n-2+\sum_{i=1}^{n} \operatorname{deg}\left(x_{i}\right)
$$


These $\ell_{n}$ define an $\mathrm{L}_{\infty}$ algebra, if they satisfy the infinitely many relations

$$
\begin{aligned}
\mathcal{J}_{n}\left(x_{1}, \ldots, x_{n}\right):= & \sum_{i+j=n+1}(-1)^{i(j-1)} \sum_{\sigma} \chi(\sigma ; x) \\
& \ell_{j}\left(\ell_{i}\left(x_{\sigma(1)}, \ldots, x_{\sigma(i)}\right), x_{\sigma(i+1)}, \ldots, x_{\sigma(n)}\right)=0 .
\end{aligned}
$$

The permutations are restricted to the ones with

$$
\sigma(1)<\cdots<\sigma(i), \quad \sigma(i+1)<\cdots<\sigma(n),
$$

and the sign $\chi(\sigma ; x)= \pm 1$ can be read off from (2.2). The first relations $\mathcal{J}_{n}$ with $n=1,2,3, \ldots$ can be schematically written as

$$
\begin{aligned}
& \mathcal{J}_{1}=\ell_{1} \ell_{1}, \quad \mathcal{J}_{2}=\ell_{1} \ell_{2}-\ell_{2} \ell_{1}, \quad \mathcal{J}_{3}=\ell_{1} \ell_{3}+\ell_{2} \ell_{2}+\ell_{3} \ell_{1}, \\
& \mathcal{J}_{4}=\ell_{1} \ell_{4}-\ell_{2} \ell_{3}+\ell_{3} \ell_{2}-\ell_{4} \ell_{1},
\end{aligned}
$$

from which one can deduce the scheme for the higher $\mathcal{J}_{n}$. More concretely, the first $\mathrm{L}_{\infty}$ relations read

$$
\begin{aligned}
\ell_{1}\left(\ell_{1}(x)\right) & =0 \\
\ell_{1}\left(\ell_{2}\left(x_{1}, x_{2}\right)\right) & =\ell_{2}\left(\ell_{1}\left(x_{1}\right), x_{2}\right)+(-1)^{x_{1}} \ell_{2}\left(x_{1}, \ell_{1}\left(x_{2}\right)\right),
\end{aligned}
$$

revealing that $\ell_{1}$ must be a nilpotent derivation with respect to $\ell_{2}$. Denoting $(-1)^{x_{i}}=(-1)^{\operatorname{deg}\left(x_{i}\right)}$ the full relation $\mathcal{J}_{3}$ reads

$$
\begin{aligned}
0= & \ell_{1}\left(\ell_{3}\left(x_{1}, x_{2}, x_{3}\right)\right)+ \\
& \ell_{2}\left(\ell_{2}\left(x_{1}, x_{2}\right), x_{3}\right)+(-1)^{\left(x_{2}+x_{3}\right) x_{1}} \ell_{2}\left(\ell_{2}\left(x_{2}, x_{3}\right), x_{1}\right)+ \\
& (-1)^{\left(x_{1}+x_{2}\right) x_{3}} \ell_{2}\left(\ell_{2}\left(x_{3}, x_{1}\right), x_{2}\right)+ \\
& \ell_{3}\left(\ell_{1}\left(x_{1}\right), x_{2}, x_{3}\right)+(-1)^{x_{1}} \ell_{3}\left(x_{1}, \ell_{1}\left(x_{2}\right), x_{3}\right)+(-1)^{x_{1}+x_{2}} \ell_{3}\left(x_{1}, x_{2}, \ell_{1}\left(x_{3}\right)\right)
\end{aligned}
$$

and means that the Jacobi identity for the $\ell_{2}$ product is mildly violated by $\ell_{1}$ exact expressions. For this reason, $\mathrm{L}_{\infty}$ algebras are also called strong homotopy Lie algebras in the mathematical literature.

The framework of $\mathrm{L}_{\infty}$ algebras is quite flexible and it has been suggested that every classical perturbative gauge theory (derived from string theory), including its dynamics, is organized by an underlying $\mathrm{L}_{\infty}$ structure [8]. For sure, the pure gauge algebra of such theories satisfies the $\mathrm{L}_{\infty}$ identities. To see this, let us assume that the field theory has a standard type gauge structure, meaning that the variations of the fields can be organized unambiguously into a sum of terms each of a definite power in the fields. Defining the space of gauge parameters $\varepsilon$ to be $X_{0}$ and the field space $\Phi$ to be $X_{-1}$ and setting all other graded vector spaces to be trivial, the gauge variations can be expressed as

$$
\delta_{\varepsilon} \Phi=\sum_{n \geq 0} \frac{1}{n !}(-1)^{\frac{n(n-1)}{2}} \ell_{n+1}(\varepsilon, \underbrace{\Phi, \ldots, \Phi}_{n \text { times }}) .
$$

It was shown in $[2,5,8,12]$, that the closure of the symmetry variations

$$
\left[\delta_{\varepsilon_{1}}, \delta_{\varepsilon_{2}}\right] \Phi=\delta_{-C\left(\varepsilon_{1}, \varepsilon_{2}, \Phi\right)} \Phi
$$


and the Jacobi identity

$$
\sum_{\text {cycl }}\left[\delta_{\varepsilon_{1}},\left[\delta_{\varepsilon_{2}}, \delta_{\varepsilon_{3}}\right]\right]=0
$$

are equivalent to the $\mathrm{L}_{\infty}$ relations with two and three gauge parameters. Here the closure relation allows for a field dependent gauge parameter which can be written in terms of $\mathrm{L}_{\infty}$ products as

$$
C\left(\varepsilon_{1}, \varepsilon_{2}, \Phi\right)=\sum_{n \geq 0} \frac{1}{n !}(-1)^{\frac{n(n-1)}{2}} \ell_{n+2}(\varepsilon_{1}, \varepsilon_{2}, \underbrace{\Phi, \ldots, \Phi}_{n \text { times }}) .
$$

Since it is precisely these relations that we will extend to the quantum case, let us briefly exemplify the procedure of identifying the constraints arising from the gauge closure with $\mathrm{L}_{\infty}$ relations up to cubic order in the fields. Using (2.9), the gauge commutator reads

$$
\begin{aligned}
{\left[\delta_{\varepsilon_{1}}, \delta_{\varepsilon_{2}}\right] \Phi=} & \left\{\ell_{2}\left(\varepsilon_{2}, \ell_{1}\left(\varepsilon_{1}\right)\right)\right. \\
& +\ell_{2}\left(\varepsilon_{2}, \ell_{2}\left(\varepsilon_{1}, \Phi\right)\right)-\ell_{3}\left(\varepsilon_{2}, \ell_{1}\left(\varepsilon_{1}\right), \Phi\right) \\
& \left.-\ell_{3}\left(\varepsilon_{2}, \ell_{2}\left(\varepsilon_{1}, \Phi\right), \Phi\right)-\frac{1}{2} \ell_{2}\left(\varepsilon_{2}, \ell_{3}\left(\varepsilon_{1}, \Phi, \Phi\right)\right)\right\} \\
& -\left\{\varepsilon_{1} \leftrightarrow \varepsilon_{2}\right\}+\mathcal{O}\left(\Phi^{3}\right),
\end{aligned}
$$

while the right hand side of the gauge closure condition can be expanded as

$$
\begin{aligned}
\delta_{-C\left(\varepsilon_{1}, \varepsilon_{2}, \Phi\right)} \Phi= & \delta_{-\ell_{2}\left(\varepsilon_{1}, \varepsilon_{2}\right)} \Phi+\delta_{-\ell_{3}\left(\varepsilon_{1}, \varepsilon_{2}, \Phi\right)} \Phi+\delta_{\frac{1}{2} \ell_{4}\left(\varepsilon_{1}, \varepsilon_{2}, \Phi, \Phi\right)} \Phi+\mathcal{O}\left(\Phi^{3}\right) \\
= & -\ell_{1}\left(\ell_{2}\left(\varepsilon_{1}, \varepsilon_{2}\right)\right)-\ell_{2}\left(\ell_{2}\left(\varepsilon_{1}, \varepsilon_{2}\right), \Phi\right)+\frac{1}{2} \ell_{3}\left(\ell_{2}\left(\varepsilon_{1}, \varepsilon_{2}\right), \Phi, \Phi\right) \\
& -\ell_{1}\left(\ell_{3}\left(\varepsilon_{1}, \varepsilon_{2}, \Phi\right)\right)-\ell_{2}\left(\ell_{3}\left(\varepsilon_{1}, \varepsilon_{2}, \Phi\right), \Phi\right) \\
& +\frac{1}{2} \ell_{1}\left(\ell_{4}\left(\varepsilon_{1}, \varepsilon_{2}, \Phi, \Phi\right)\right)+\mathcal{O}\left(\Phi^{3}\right) .
\end{aligned}
$$

Comparing (2.14) with (2.13) we see that demanding closure yields conditions on the $\ell_{n}$ products. For instance, at zeroth order in $\Phi$ one obtains the condition

$$
\ell_{1}\left(\ell_{2}\left(\varepsilon_{1}, \varepsilon_{2}\right)\right)=\ell_{2}\left(\varepsilon_{1}, \ell_{1}\left(\varepsilon_{2}\right)\right)-\ell_{2}\left(\varepsilon_{2}, \ell_{1}\left(\varepsilon_{1}\right)\right) .
$$

Upon interchanging the arguments this is exactly the $\mathrm{L}_{\infty}$ relation $\mathcal{J}_{2}\left(\varepsilon_{1}, \varepsilon_{2}\right)=0$ in $(2.7)$. At first order in $\Phi$ one gets

$$
\begin{aligned}
0= & \ell_{2}\left(\varepsilon_{2}, \ell_{2}\left(\varepsilon_{1}, \Phi\right)\right)+\ell_{2}\left(\ell_{2}\left(\varepsilon_{1}, \varepsilon_{2}\right), \Phi\right)-\ell_{2}\left(\varepsilon_{1}, \ell_{2}\left(\varepsilon_{2}, \Phi\right)\right) \\
& -\ell_{3}\left(\varepsilon_{2}, \ell_{1}\left(\varepsilon_{1}\right), \Phi\right)+\ell_{3}\left(\varepsilon_{1}, \ell_{1}\left(\varepsilon_{2}\right), \Phi\right) \\
& +\ell_{1}\left(\ell_{3}\left(\varepsilon_{1}, \varepsilon_{2}, \Phi\right)\right) .
\end{aligned}
$$

This is the $\mathrm{L}_{\infty}$ relation $\mathcal{J}_{3}\left(\varepsilon_{1}, \varepsilon_{2}, \Phi\right)=0$ in which the term $\ell_{3}\left(\varepsilon_{1}, \epsilon_{2}, \ell_{1}(\Phi)\right)$ is missing, as we have set $X_{-2}=0$. This result is just a consequence of the general two relations between 
the classical gauge algebra and the $\mathrm{L}_{\infty}$ algebra:

$$
\begin{aligned}
& \text { gauge closure } \Leftrightarrow 0=\mathcal{J}_{n}(\varepsilon_{1}, \varepsilon_{2}, \underbrace{\Phi, \ldots, \Phi}_{n-2 \text { times }}), \\
& \text { gauge Jacobi identity } \Leftrightarrow 0=\mathcal{J}_{n}(\varepsilon_{1}, \varepsilon_{2}, \varepsilon_{3}, \underbrace{\Phi, \ldots, \Phi}_{n-3 \text { times }}) .
\end{aligned}
$$

As one can check, these are actually the only non-trivial $\mathrm{L}_{\infty}$ relations in case that the graded vector space is given by $X=X_{0} \oplus X_{-1}$. This can be generalized by adding a vector space $X_{-2}$ containing the equations of motion, thus allowing the freedom that gauge closure only holds on-shell [8].

\section{Quantum $\mathrm{L}_{\infty}$ gauge algebras}

In the last section we recalled how the $\mathrm{L}_{\infty}$ relations guarantee the consistency of a classical gauge algebra. Recently it was shown that also global classical $\mathcal{W}$ algebras arising in twodimensional conformal field theory yield non-trivial examples of $\mathrm{L}_{\infty}$ algebras. Driven by the aim to extract physically well motivated aspects of a quantum extension of $\mathrm{L}_{\infty}$ algebras, we analyze whether a generalized version of this correspondence holds for quantum $\mathcal{W}$ algebras. On the way, we encounter a couple of new structures that can be traced back to the non-associativity of the normal ordered products appearing in the quantum $\mathcal{W}$ algebra. Resolving these issues guides us to a proposal of a quantum $\mathrm{L}_{\infty}$ gauge algebra that we will present in the section.

Concretely, in section 3.1, by demanding consistency of the quantized symmetry algebra, we outline how the usual notion of an $\mathrm{L}_{\infty}$ algebra has to be adjusted for a quantum $\mathrm{L}_{\infty}$ algebra. We find that beyond the higher products also the $\mathrm{L}_{\infty}$ relations receive quantum corrections, whose origin lies in the necessity to perform Wick contractions between quantum fields.

In 3.2 we review the $\mathrm{L}_{\infty}$ algebra of closed string field theory and the quantum corrections appearing there. As it turns out, the quantum corrections due to Wick contractions do not appear there.

\subsection{The quantum $\mathrm{L}_{\infty}$ algebra of a quantum symmetry}

Going from a classical field theory to a quantum field theory, the fields become operator valued. We want to consider quantum symmetries which in the classical limit $\hbar \rightarrow 0$ become a classical symmetry of the kind described in the last section. In particular we are still working only on the graded vector space $X=X_{0} \oplus X_{-1}$, where the symmetry parameters are contained in $X_{0}$ and the field operators in $X_{-1}$. In the case of $\mathcal{W}$-algebras, the infinitely many symmetry parameters ${ }^{1}$ are compactly encoded in $\epsilon(z)=\sum_{n \in \mathbb{Z}} z^{n+\Delta-1} \epsilon_{n}$ and the infinitely many symmetry generators in $W(z)=\sum_{n \in \mathbb{Z}} z^{-n-\Delta} W_{n}$. Here $\Delta$ denotes the conformal dimension of the chiral field $W(z)$.

\footnotetext{
${ }^{1}$ Note that the holomorphic function $\epsilon(z)$ does not parametrize a gauge variation, as the latter would depend on $z$ and $\bar{z}$.
} 
In the classical case it was crucial that the variation of the field could be organized in terms of definite powers in the fields to define the corresponding $\mathrm{L}_{\infty}$ products. In order to adapt the notion of field powers, we have to specify an operator product in the quantum case.

Inspired by the analysis of $\mathcal{W}$ algebras, to be discussed in detail in section 4 , we define the operator product to be the symmetrized normal ordered product

$$
A \star B=\frac{1}{2}(N(A B)+N(B A)) .
$$

This is a convenient choice, as by taking the classical limit $\hbar \rightarrow 0$, it becomes the usual point-wise multiplication of fields. Let us already point out one subtlety relative to the classical case, that will be one source of quantum corrections. As can be seen from the notion of the normal ordering in $2 \mathrm{~d} \mathrm{CFT}$, the $\star$ product above while commutative fails to be associative. There ${ }^{2}$ the non-associativity of the normal ordered product is given by

$$
(\varepsilon A) \star B-\varepsilon(A \star B)=\varepsilon(\stackrel{\curvearrowleft}{A B}),
$$

where $\varepsilon$ is just a c-number symmetry variation and $A, B$ are operator valued fields. Moreover, the last term denotes extra terms arising from the contraction between the two operators defined as

$$
\lim _{y \rightarrow x}(A(x) B(y)-(\stackrel{\sqcap}{A B})(x, y))=N(A B)(x)
$$

which in a CFT is nothing else than the singular part of the operator product expansion. Having defined the product between operators, we assume that variations of the field can be schematically written in the form

$$
\delta_{\varepsilon}^{\text {qu }} \Phi \sim \sum_{n} \varepsilon \underbrace{\Phi \star \cdots \star \Phi}_{n \text { times }}
$$

where for simplicity we considered bosonic fields and symmetry parameters. Following the lines of the classical discussion we define graded symmetric multilinear quantum n-products

$$
L_{n+1}: X^{\otimes n} \rightarrow X
$$

and rewrite the variation in the form

$$
\delta_{\varepsilon}^{\mathrm{qu}} \Phi=\sum_{n \geq 0} \frac{1}{n !}(-1)^{\frac{n(n-1)}{2}} L_{n+1}(\varepsilon, \underbrace{\Phi, \ldots, \Phi}_{n \text { times }}) .
$$

The quantum $L_{n}$ products still carry the intrinsic grading $\operatorname{deg} L_{n}=n-2$. Since the starproduct is symmetric, the $L$-products are automatically symmetric when interchanging two fields. Since in the limit $\hbar \rightarrow 0$, the star product becomes the normal field product, the quantum $L_{n}$-products will become the classical $\ell_{n}$-products with the right degree and symmetry properties.

\footnotetext{
${ }^{2}$ This can be shown using the general formula 6.227 in [13].
} 
Following the classical analysis, the question now is which constraints arise from demanding the closure of the quantum symmetry algebra

$$
\left[\delta_{\varepsilon_{1}}^{\mathrm{qu}}, \delta_{\varepsilon_{2}}^{\mathrm{qu}}\right] \Phi=\delta_{-C\left(\varepsilon_{1}, \varepsilon_{2}, \Phi\right)}^{\mathrm{qu}} \Phi
$$

and the Jacobi identity

$$
\sum_{\text {cycl }}\left[\delta_{\varepsilon_{1}}^{\mathrm{qu}},\left[\delta_{\varepsilon_{2}}^{\mathrm{qu}}, \delta_{\varepsilon_{3}}^{\mathrm{qu}}\right]\right]=0 .
$$

Here, the field dependent closure parameter $C\left(\varepsilon_{1}, \varepsilon_{2}, \Phi\right)$ should still be expressed in terms of the symmetrized normal ordered product

$$
C\left(\varepsilon_{1}, \varepsilon_{2}, \Phi\right) \sim \sum_{n} \varepsilon_{1} \varepsilon_{2} \cdot \Phi \star \cdots \star \Phi
$$

allowing to read off the $L_{n}$ products with two symmetry parameters

$$
C\left(\varepsilon_{1}, \varepsilon_{2}, \Phi\right)=\sum_{n \geq 0} \frac{1}{n !}(-1)^{\frac{n(n-1)}{2}} L_{n+2}(\varepsilon_{1}, \varepsilon_{2}, \underbrace{\Phi, \ldots, \Phi}_{n \text { times }}) .
$$

To identify potential sources of quantum corrections in the $\mathrm{L}_{\infty}$ relations, we write out the first few terms of both sides of the closure condition (3.7). Up to second order in the fields, the left hand side can be expanded as

$$
\begin{aligned}
{\left[\delta_{\varepsilon_{1}}^{\mathrm{qu}}, \delta_{\varepsilon_{2}}^{\mathrm{qu}}\right] \Phi=} & \left\{L_{2}\left(\varepsilon_{2}, L_{1}\left(\varepsilon_{1}\right)\right)\right. \\
& +L_{2}\left(\varepsilon_{2}, L_{2}\left(\varepsilon_{1}, \Phi\right)\right)-L_{3}\left(\varepsilon_{2}, L_{1}\left(\varepsilon_{1}\right), \Phi\right) \\
& \left.-L_{3}\left(\varepsilon_{2}, L_{2}\left(\varepsilon_{1}, \Phi\right), \Phi\right)-\frac{1}{2} L_{2}\left(\varepsilon_{2}, L_{3}\left(\varepsilon_{1}, \Phi, \Phi\right)\right)\right\} \\
& -\left\{\varepsilon_{1} \leftrightarrow \varepsilon_{2}\right\},
\end{aligned}
$$

while the right side is

$$
\begin{aligned}
\delta_{-C^{\mathrm{qu}}\left(\varepsilon_{1}, \varepsilon_{2}, \Phi\right)} \Phi= & \delta_{-L_{2}\left(\varepsilon_{1}, \varepsilon_{2}\right)} \Phi+\delta_{-L_{3}\left(\varepsilon_{1}, \varepsilon_{2}, \Phi\right)} \Phi \\
= & -L_{1}\left(L_{2}\left(\varepsilon_{1}, \varepsilon_{2}\right)\right)-L_{2}\left(L_{2}\left(\varepsilon_{1}, \varepsilon_{2}\right), \Phi\right)+\frac{1}{2} L_{3}\left(L_{2}\left(\varepsilon_{1}, \varepsilon_{2}\right), \Phi, \Phi\right) \\
& -L_{1}\left(L_{3}\left(\varepsilon_{1}, \varepsilon_{2}, \Phi\right)\right)-L_{2}\left(L_{3}\left(\varepsilon_{1}, \varepsilon_{2}, \Phi\right), \Phi\right) .
\end{aligned}
$$

To read off the quantum $\mathrm{L}_{\infty}$ relations, we now sort (3.11) and (3.12) according to the power in $\Phi$. Since now the power of $\Phi$ is with respect to the symmetrized normal ordered product, this is a bit more subtle than in the classical case. One first has to bring all terms into the schematic form $\left(\varepsilon_{1} \varepsilon_{2}\right) \cdot(\Phi \star \cdots \star \Phi)$ that also appeared in the definitions of the $L$-products (3.6) and (3.10). While some terms are already of this form, for others a rebracketing is necessary.

Consider for instance the fourth term in (3.11) that, upon using (3.4), can be schematically written as

$$
L_{3}\left(\varepsilon_{2}, L_{2}\left(\varepsilon_{1}, \Phi\right), \Phi\right) \sim \varepsilon_{2}\left(\left(\varepsilon_{1} \Phi\right) \star \Phi\right) .
$$


Using the non-associativity of the $\star$-product (3.2), this becomes

$$
L_{3}\left(\varepsilon_{2}, L_{2}\left(\varepsilon_{1}, \Phi\right), \Phi\right)=\varepsilon_{1} \varepsilon_{2} \cdot(\Phi \star \Phi)+\varepsilon_{1} \varepsilon_{2} \cdot(\Phi \Phi) .
$$

Let us assume for simplicity a free theory such that $\Phi \Phi$ is proportional $\hbar \mathbf{1}$. Then the last term in (3.14) is proportional to $\epsilon_{1}$ and $\epsilon_{2}$ and therefore a quantum correction to the $\mathrm{L}_{\infty}$ relation at zeroth order in $\Phi$. Treating the last term in (3.12) in an analogous way, we find the quantum corrected $\mathrm{L}_{\infty}$ relation at zeroth order in $\Phi$

$$
\begin{aligned}
0= & L_{2}\left(L_{1}\left(\varepsilon_{1}\right), \varepsilon_{2}\right)+L_{2}\left(\varepsilon_{1}, L_{1}\left(\varepsilon_{2}\right)\right)+L_{1}\left(L_{2}\left(\varepsilon_{1}, \varepsilon_{2}\right)\right) \\
& -L_{3}\left(\varepsilon_{2}, L_{2}\left(\varepsilon_{1}, \Phi\right), \Phi\right)+L_{3}\left(\varepsilon_{1}, L_{2}\left(\varepsilon_{2}, \Phi\right), \Phi\right)+L_{2}\left(L_{3}\left(\varepsilon_{1}, \varepsilon_{2}, \Phi\right), \Phi\right) .
\end{aligned}
$$

Similarly also all other $\mathrm{L}_{\infty}$ relations get corrected by contractions of higher $\mathrm{L}_{\infty}$ relations.

Let us summarize: guided by quantum algebras in $2 \mathrm{~d}$ CFT, we identified two sources of quantum corrections to $\mathrm{L}_{\infty}$ algebra. First, relative to the classical products, the higher quantum $\mathrm{L}_{\infty}$ products can receive corrections of higher order in $\hbar$. The second kind of quantum corrections arises from contractions between quantum fields that appear when sorting the relations in powers of the field. These contractions change the power of the fields so that the classically separated $\mathrm{L}_{\infty}$ relations receive quantum suppressed off-diagonal corrections.

We want to stress that the contractions differ severely from theory to theory. While in free theories the contraction is proportional to the identity operator, in interacting theories (like generic CFTs) the contraction of two fields is usually field dependent again. We can therefore not provide a general closed formula for which contraction of which $\mathrm{L}_{\infty}$ relation contributes to which other $\mathrm{L}_{\infty}$ relation.

Guided by these observations we suggest to define quantum $\mathrm{L}_{\infty}$ algebras that govern (global) quantum symmetries as follows: one has a graded vector space $X=X_{0} \oplus X_{-1}$, where $X_{n}$ is said to have degree $n$. In addition there are multi-linear quantum products $L_{n}\left(x_{1}, \ldots, x_{n}\right)$ that have degree $\operatorname{deg}\left(L_{n}\right)=n-2$ so that

$$
\operatorname{deg}\left(L_{n}\left(x_{1}, \ldots, x_{n}\right)\right)=n-2+\sum_{i=1}^{n} \operatorname{deg}\left(x_{i}\right) .
$$

Each product can in principle receive quantum corrections at any power in $\hbar$. The products are graded commutative, i.e.

$$
L_{n}\left(\ldots, x_{1}, x_{2}, \ldots\right)=(-1)^{1+\operatorname{deg}\left(x_{1}\right) \operatorname{deg}\left(x_{2}\right)} L_{n}\left(\ldots, x_{2}, x_{1}, \ldots\right)
$$

Like in the classical case, one defines

$$
\begin{aligned}
\mathcal{J}_{n}^{\mathrm{qu}}\left(x_{1}, \ldots, x_{n}\right):=\sum_{i+j=n+1}(-1)^{i(j-1)} \sum_{\sigma} \chi(\sigma ; x) \\
L_{j}\left(L_{i}\left(x_{\sigma(1)}, \ldots, x_{\sigma(i)}\right), x_{\sigma(i+1)}, \ldots, x_{\sigma(n)}\right) .
\end{aligned}
$$


The $L_{n}$ products define a quantum $\mathrm{L}_{\infty}$ algebra if they satisfy for each $m=2,3$ and $n \in \mathbb{Z}_{0}^{+}$

$$
\mathcal{J}_{m+n}^{\mathrm{qu}}\left(\epsilon_{1}, \ldots, \epsilon_{m}, x_{1}, \ldots, x_{n}\right)+\sum_{\substack{\left(y_{1}, \ldots, y_{k}\right) \\ \rightarrow\left(x_{1}, \ldots, x_{n}\right)}} \hbar^{\xi} \mathcal{J}_{m+k}^{\mathrm{qu}}(\epsilon_{1}, \ldots, \epsilon_{m}, \underbrace{y_{1}, \ldots, y_{k}}_{\rightarrow\left(x_{1}, \ldots, x_{n}\right)})=0 .
$$

Since this is the main formula of the paper we want to explain the formula in more detail. $\epsilon_{i} \in X_{0}$ is a symmetry parameter and $x_{i} \in X_{-1}$ is a field. While the first term is the known one from the classical $\mathrm{L}_{\infty}$ relations, the second term contains the crucial new feature of quantum $\mathrm{L}_{\infty}$ algebras, namely the corrections due to contractions of other $\mathrm{L}_{\infty}$ relations. To cover all such corrections we sum over all $\mathrm{L}_{\infty}$ relations whose field input $\left(y_{1}, \ldots, y_{k}\right)$ can contract into $\left(x_{1}, \ldots, x_{n}\right)$. The $\xi \geq 1$ counts the number of contractions employed to convert the dependence on $\left(y_{1}, \ldots, y_{m}\right)$ into a dependence on $\left(x_{1}, \ldots, x_{n}\right)$. The underbrace signals that only the terms that arise from the particular contraction are to be taken here. To avoid permutation factors we let the sum run only over $\left(y_{1}, \ldots, y_{k}\right)$ that are not equal under permutation. Furthermore notice that the order of the $\left(y_{1}, \ldots, y_{k}\right)$ does not play a role since the $\mathcal{J}^{\text {qu }}$ share the permutation property of $(3.17) .^{3}$

Let us provide a more general and mathematically precise definition for the quantum $\mathrm{L}_{\infty}$ algebra. Since the quantum corrections mix the different $\mathrm{L}_{\infty}$ relations, we can also define quantum $\mathrm{L}_{\infty}$ algebras very compactly by demanding that for $m \in\{2,3\}$ and $\epsilon_{i} \in X_{0}$ the sum of all $\mathrm{L}_{\infty}$ relations vanish

$$
\sum_{n=1}^{\infty} \sum_{\left(x_{1}, \ldots x_{n}\right) \in X_{-1}^{n}} \mathcal{J}_{m+n}^{\mathrm{qu}}\left(\epsilon_{1}, \ldots, \epsilon_{m}, x_{1}, \ldots x_{n}\right)=0,
$$

where as before the second sum runs only over distinct $\left(x_{1}, \ldots, x_{n}\right)$. In case the $\mathrm{L}$ products do not change the power of the input, the terms in (3.20) separate into the classical $\mathrm{L}_{\infty}$ relations (2.4). On the other hand, using normal ordered products in the $\mathrm{L}$ products, (3.20) reduces to the former definition (3.19). Nevertheless we want to stress that in general (3.20) does not need any physical input in form of a contraction. From the mathematical viewpoint the definition (3.20) might therefore be more appealing. We nevertheless prefer (3.19) that also makes it manifest that in the $\hbar \rightarrow 0$ limit one encounters the classical $\mathrm{L}_{\infty}$ relations and that their off-diagonal quantum corrections arise from the contraction of quantum fields.

In section 4 we show in much detail how quantum $\mathcal{W}$ algebras fit precisely into this definition of quantum $\mathrm{L}_{\infty}$ algebras. Especially in section 4.4 we will demonstrate that the quantum relations (3.19) can be given a precise meaning for the quantum $\mathcal{W}_{3}$ algebra.

\subsection{Comparison to the $\mathrm{L}_{\infty}$ algebra of CSFT}

We will now compare our definition for a quantum $\mathrm{L}_{\infty}$ algebra with the $\mathrm{L}_{\infty}$ algebra of closed string field theory (CSFT) $[1,11]$. To distinguish these two different $\mathrm{L}_{\infty}$ definitions,

\footnotetext{
${ }^{3}$ Here an obstacle becomes apparent if one tries to generalize the above definition beyond the given case where contractions appear only between elements of $X_{-1}$. When contractions appear not only between elements with even parity the order of the $y_{1}, \ldots, y_{k}$ does indeed matter. Lacking an example to follow we cannot give a precise ordering prescription to fix this issue here.
} 
we will follow Markl [11] and call the $\mathrm{L}_{\infty}$ algebra of CSFT a loop $\mathrm{L}_{\infty}$ algebra, while the definition from last section will be called quantum $\mathrm{L}_{\infty}$ algebra.

In a loop $\mathrm{L}_{\infty}$ algebras one usually expands the quantum products according to their loop level, thus their power of $\hbar$

$$
L_{n}\left(x_{1}, \ldots, x_{n}\right)=\sum_{g} L_{n}^{g}\left(x_{1}, \ldots x_{n}\right),
$$

where $L_{n}^{g}$ is proportional to $\hbar^{g}$. Then, the $L_{n}^{g}$ products define a loop $\mathrm{L}_{\infty}$ algebra, if for any level $g$ the following relation holds (we use the notation of [11])

$$
\begin{aligned}
0= & \sum_{g_{1}+g_{2}=g} \sum_{i+j=n+1}(-1)^{i(j-1)} \sum_{\sigma} \chi(\sigma ; x) \\
& \quad \times L_{j}^{g_{1}}\left(L_{i}^{g_{2}}\left(x_{\sigma(1)}, \ldots, x_{\sigma(i)}\right), x_{\sigma(i+1)}, \ldots, x_{\sigma(n)}\right) \\
& +\frac{1}{2} \sum_{s}(-1)^{\operatorname{deg}\left(h_{s}\right)+n-g} L_{n+2}^{g-1}\left(h_{s}, h^{s}, x_{1}, \ldots, x_{n}\right) .
\end{aligned}
$$

The sum over $s$ in the last term runs over a basis of fields labeled by $s$. The field with an upper index, $h^{s}$, is the conjugate field to $h_{s}$ with respect to a scalar product $\left\langle h^{s}, h_{t}\right\rangle=\delta_{t}^{s}$. The $\sum_{s} L_{n}^{g-1}\left(h_{s}, h^{s}, \ldots\right)$ can be interpreted as an identity operator. When contracting $h_{s}, h^{s}$ to eliminate this identity operator, we obtain an additional $\hbar$ factor such that, together with the $\hbar^{g-1}$ from the $L_{n}^{g-1}$, the last term is proportional to $\hbar^{g}$ as well.

Let us compare the defining relations of (global) quantum and (gauge) loop $\mathrm{L}_{\infty}$ algebras: the first part of the loop $\mathrm{L}_{\infty}$ relation (3.22) appears in quantum $\mathrm{L}_{\infty}$ algebras as the order $\hbar^{g}$ term, when inserting the expansion (3.21) into the first term of (3.19). The second term of (3.22) does not appear in the quantum $\mathrm{L}_{\infty}$ relations in (3.19). The reason for this is, that the quantum $\mathrm{L}_{\infty}$ was derived in a setting where the total vector space contained only degree 0 objects, the symmetry parameters, and degree -1 objects, the fields. Therefore $X=X_{0} \oplus X_{-1}$ and all objects with a degree other than 0 and -1 were set to zero. Demanding all terms in the defining relation of loop $\mathrm{L}_{\infty}$ algebras (3.22) to have the same degree, we find

$$
\operatorname{deg}\left(h_{s}\right)+\operatorname{deg}\left(h^{s}\right)=-3 .
$$

Since $h_{s}$ is a field, its degree is $\operatorname{deg}\left(h_{s}\right)=-1$ and the degree of $h^{s}$ is bound to be $\operatorname{deg}\left(h^{s}\right)=-2$. Therefore, $h^{s}$ is trivial and the second term in (3.22) could not appear in the derivation of the quantum $\mathrm{L}_{\infty}$ based entirely on quantum gauge variations.

Remarkably, the second term in the quantum $\mathrm{L}_{\infty}$ relation (3.19) has no counterpart in the loop $\mathrm{L}_{\infty}$ algebras. Therefore the $\mathrm{L}_{\infty}$ relations of the CSFT $\mathrm{L}_{\infty}$ algebra do not receive corrections from contraction terms. The question arises if there exist a connection between the two definitions. From the current status, the answer is not completely clear to us and more work or insight is required to fully clarify it. We can only say that the structure of (gauge) loop $\mathrm{L}_{\infty}$ arose as a consequence of the quantum master equation of the BV-formalism for the CSFT quantum action. On the contrary, our (global) quantum $\mathrm{L}_{\infty}$ definition is based on the analysis of bootstrapped and therefore exactly solvable global quantum $\mathcal{W}$ algebras in $2 \mathrm{~d}$ CFT. 


\section{The quantum $\mathcal{W}_{3}-\mathrm{L}_{\infty}$ algebra}

In the recent paper [9] it was shown that (classical) $\mathcal{W}$ algebras are highly non-trivial (classical) $\mathrm{L}_{\infty}$ algebras with field dependent symmetry parameters. In this section we will show that the quantum $\mathcal{W}_{3}$-algebra fits into the framework of the quantum $\mathrm{L}_{\infty}$ algebra of section 3.1 (and was in fact motivating it). We expect that more general quantum $\mathcal{W}$-algebras will even provide more intricate examples of quantum $\mathrm{L}_{\infty}$ algebras.

\section{1 $\mathcal{W}$ algebras}

In two-dimensional conformal field theories the energy momentum tensor $T(z)$ is a quasi primary field that has conformal dimension two, generates the conformal transformations and obeys the Virasoro algebra. A $\mathcal{W}$ algebra is an extension of the Virasoro algebra by chiral primary fields of conformal dimension usually larger than two. The prototype example is Zamolodchikov's $\mathcal{W}_{3}$ algebra [14], generated by two fields $\{T(z), W(z)\}$ of conformal dimensions two and three. The (quantum) OPEs among these fields are known to be ${ }^{4}$

$$
\begin{aligned}
\frac{1}{\hbar} T(z) \circ T(w)= & \frac{c / 2}{(z-w)^{4}}+2\left(\frac{T(w)}{(z-w)^{2}}+\frac{1}{2} \frac{\partial T(w)}{(z-w)}\right), \\
\frac{1}{\hbar} T(z) \circ W(w)= & 3\left(\frac{W(w)}{(z-w)^{2}}+\frac{1}{3} \frac{\partial W(w)}{(z-w)}\right), \\
\frac{1}{\hbar} W(z) \circ W(w)= & \frac{c / 3}{(z-w)^{6}} \\
& +\alpha\left(\frac{T(w)}{(z-w)^{4}}+\frac{1}{2} \frac{\partial T(w)}{(z-w)^{3}}+\frac{3}{20} \frac{\partial^{2} T(w)}{(z-w)^{2}}+\frac{1}{30} \frac{\partial^{3} T(w)}{(z-w)}\right) \\
& +\beta\left(\frac{\Lambda^{\mathrm{qu}}(w)}{(z-w)^{2}}+\frac{1}{2} \frac{\partial \Lambda^{\mathrm{qu}}(w)}{(z-w)}\right) .
\end{aligned}
$$

Here the field $\Lambda^{\text {qu }}$ denotes the normal ordered product

$$
\Lambda^{\mathrm{qu}}=N(T T)-\hbar \frac{3}{10} \partial^{2} T
$$

where we have indicated the quantum correction linear in $T$. The corresponding algebra for the modes satisfies the Jacobi-identity for

$$
\alpha=2, \quad \beta=\frac{32}{5 c+22 \hbar} .
$$

Following [17], in these formulas we have introduced $\hbar$ so that the classical limit and its quantum corrections are clearly visible. In the $\hbar \rightarrow 0$ limit, the commutator (singular part of the OPE) becomes the Poisson bracket

$$
\{\cdot, \cdot\}_{\mathrm{PB}}=\lim _{\hbar \rightarrow 0} \frac{1}{i \hbar}[\cdot, \cdot] .
$$

\footnotetext{
${ }^{4} \mathrm{Up}$ to some structure constants, the form of the OPE between quasi-primary fields is generally known [15] (for a pedestrian derivation see also [16]), as has been exploited for the classical $\mathcal{W}-\mathrm{L}_{\infty}$ algebra relation in [9].
} 
There exist three sources of quantum corrections. Two of them are manifest in the $\hbar$ corrections in (4.2) and $(4.3)^{5}$ and the third is the appearance of the normal ordered product $N(T T)$ instead of the usual point-wise multiplication $(T T)$ in the classical case.

The normal ordered product between two chiral fields is defined as

$$
N(\phi \chi)(w)=\frac{1}{2 \pi i} \oint_{\gamma(w)} d z \frac{\phi(z) \circ \chi(w)}{(z-w)}
$$

where $\gamma(w)$ is a path encircling $w$ counterclockwise once. The normal ordered product is therefore the first regular term in the OPE between the two fields. Note that this product is neither commutative nor associative. Since for the correspondence to an $\mathrm{L}_{\infty}$ algebra one needs graded symmetric products, we use the symmetrized normal ordered product $\star$ from (3.1) that is still non-associative. To demonstrate this, let us explicitly compute the left hand side of (3.2) for $A=B=T$

$$
\begin{aligned}
(\varepsilon T) \star T-\varepsilon(T \star T) & =\frac{1}{4 \pi i} \oint d z \frac{\epsilon(z) T(z) \circ T(w)}{(z-w)} \\
& =\frac{c \hbar}{96} \partial^{4} \epsilon+\frac{\hbar}{2} \partial^{2} \epsilon T+\frac{\hbar}{2} \partial \epsilon \partial T
\end{aligned}
$$

where both sides depend on $w$. Note that these corrections arise from the contraction of operators below the integral and that they are $\hbar$-suppressed relative to the leading order normal ordered products.

The extended symmetry algebra acts with

$$
\delta_{\varepsilon_{i}} W_{j}(w)=\frac{1}{2 \pi i} \oint_{\gamma(w)} d z \varepsilon_{i}(z) \frac{1}{\hbar} W_{i}(z) \circ W_{j}(w),
$$

where $i, j=\{T, W\}$. Instead of writing $\varepsilon_{T}$ and $\varepsilon_{W}$ from now on we will write $\varepsilon$ for $\varepsilon_{T}$ and $\eta$ for $\varepsilon_{W}$.

\section{2 $L_{n}$ products with one symmetry parameter}

Let us now follow the steps outlined in the sections 2 and 3.1 to construct the quantum $\mathrm{L}_{\infty}$ algebra corresponding to the quantum $\mathcal{W}_{3}$ algebra. The fields $\{T, W\}$ have degree -1 , and the symmetry parameters $\{\varepsilon, \eta\}$ have degree zero. Therefore the total vector space is $X=X_{0} \oplus X_{-1}$ and each $X_{n}=X_{n}^{T} \oplus X_{n}^{W}$ splits into a $T$ and a $W$ part. As in [9], we will use boldface to highlight vectors in this two-dimensional space, for instance $\mathbf{W}=(T, W)$ will denote either of the fields. Furthermore we equip all $L_{n}$ products with an upper index from the set $\{T, W, \epsilon, \eta\}$ that denotes in which of the four subspaces of $X$ the image of the higher product $L_{n}$ is located.

\footnotetext{
${ }^{5}$ Notice that when expanding the fraction $\beta$ we get an infinite series with terms at any order in $\hbar$. Separating the different powers of $\hbar^{g}$ in different $L_{n}^{g}$ products, as usually done in loop $\mathrm{L}_{\infty}$ algebras, see (3.21), is therefore not illuminating in this example.
} 
Inserting (4.1) in (4.7), for the quantum corrected infinitesimal variations one obtains

$$
\begin{aligned}
& \delta_{\varepsilon} T=\underbrace{\frac{c}{12} \partial^{3} \varepsilon}_{L_{1}^{T}(\varepsilon)}+\underbrace{(2 \partial \varepsilon T+\varepsilon \partial T)}_{L_{2}^{T}(\varepsilon, T)}, \\
& \delta_{\varepsilon} W=\underbrace{(3 \partial \varepsilon W+\varepsilon \partial W)}_{L_{2}^{W}(\varepsilon, W)}, \\
& \delta_{\eta} T=\underbrace{(3 \partial \eta W+2 \eta \partial W)}_{L_{2}^{T}(\eta, W)}
\end{aligned}
$$

and

$$
\begin{aligned}
\delta_{\eta} W= & \underbrace{\frac{c}{360} \partial^{5} \eta}_{L_{1}^{W}(\eta)}+\underbrace{\alpha\left(\frac{1}{6} \partial^{3} \eta T+\frac{1}{4} \partial^{2} \eta \partial T+\frac{3}{20} \partial \eta \partial^{2} T+\frac{1}{30} \eta \partial^{3} T\right)}_{L_{2}^{W}(\eta, T)} \\
& -\underbrace{\frac{3 \hbar \beta}{10}\left(\partial \eta \partial^{2} T+\frac{1}{2} \eta \partial^{3} T\right)}_{L_{2}^{W}(\eta, T)} \\
& +\underbrace{\beta\left(\partial \eta(T \star T)+\frac{1}{2} \eta \partial(T \star T)\right)}_{-\frac{1}{2} L_{3}^{W}(\eta, T, T)} .
\end{aligned}
$$

Notice that we have already written all terms in the form (3.4) such that we can directly read off the $L_{n}$ products. Compared to the classical higher products, the only change is in $\delta_{\eta} W$, where $L_{2}^{W}(\eta, T)$ receives an explicit $\hbar$-correction and $\ell_{3}^{W}(\eta, T, T)$ involves the quantum product $T \star T$.

\section{3 $L_{n}$ products with two symmetry parameters}

Recall that the $L_{n}$ products with two symmetry parameters appear in the closure condition (3.7)

$$
\left[\delta_{\varepsilon_{i}}^{\mathrm{qu}}, \delta_{\varepsilon_{j}}^{\mathrm{qu}}\right] W_{k}=\delta_{-C\left(\varepsilon_{i}, \varepsilon_{j}, \mathbf{W}\right)}^{\mathrm{qu}} W_{k}
$$

upon expanding (3.10)

$$
\mathbf{C}\left(\varepsilon_{i}, \varepsilon_{j}, \mathbf{W}\right)=\sum_{n \geq 0} \frac{1}{n !}(-1)^{\frac{n(n-1)}{2}} L_{n+2}(\varepsilon_{i}, \varepsilon_{j}, \underbrace{\mathbf{W}, \ldots, \mathbf{W}}_{n \text { times }}) .
$$

To obtain the $\mathbf{C}\left(\varepsilon_{i}, \varepsilon_{j}, \mathbf{W}\right)$ we insert (4.7) into the symmetry closure condition and use the generalized Wick theorem for chiral vertex operator algebras [18]

$$
\begin{aligned}
& \oint \frac{d y}{2 \pi i}(y-w)^{n} A(y) \circ\left(\oint \frac{d z}{2 \pi i}(z-w)^{m} B(z) \circ C(w)\right) \\
& -\oint \frac{d y}{2 \pi i}(y-w)^{m} B(y) \circ\left(\oint \frac{d z}{2 \pi i}(z-w)^{n} A(z) \circ C(w)\right) \\
& =\sum_{j=0}^{n}\left(\begin{array}{c}
n \\
j
\end{array}\right) \oint \frac{d z}{2 \pi i}\left(\oint \frac{d y}{2 \pi i}(y-z)^{j} A(y) \circ B(z)\right) \circ C(w)(z-w)^{(m+n-j)}
\end{aligned}
$$


in the special case $m, n=0$. In this way, for instance we can derive

$$
\begin{aligned}
{\left[\delta_{\varepsilon_{1}}, \delta_{\varepsilon_{2}}\right] T(z) } & =\left(\frac{1}{2 \pi i}\right)^{2} \oint d y \frac{1}{\hbar}\left(\oint d w \varepsilon_{1}(w) \varepsilon_{2}(y) \frac{1}{\hbar} T(w) \circ T(y)\right) \circ T(z) \\
& =\frac{1}{2 \pi i} \oint d y\left(\partial \varepsilon_{1}(y) \varepsilon_{2}(y)-\varepsilon_{1}(y) \partial \varepsilon_{2}(y)\right) \frac{1}{\hbar} T(y) \circ T(z),
\end{aligned}
$$

so that the C-product can be read off as

$$
\mathbf{C}\left(\varepsilon_{1}, \varepsilon_{2}, \mathbf{W}\right)=\varepsilon_{1} \partial \varepsilon_{2}-\partial \varepsilon_{1} \varepsilon_{2}:=L_{2}^{\varepsilon}\left(\varepsilon_{1}, \varepsilon_{2}\right) .
$$

Similarly we find

$$
\begin{aligned}
\mathbf{C}(\varepsilon, \eta, \mathbf{W}) & =\varepsilon \partial \eta-2 \partial \varepsilon \eta:=L_{2}^{\eta}(\varepsilon, \eta), \\
\mathbf{C}\left(\eta_{1}, \eta_{2}, \mathbf{W}\right) & =L_{2}^{\varepsilon}\left(\eta_{1}, \eta_{2}\right)+L_{3}^{\varepsilon}\left(\eta_{1}, \eta_{2}, T\right),
\end{aligned}
$$

with

$$
\begin{aligned}
L_{2}^{\varepsilon}\left(\eta_{1}, \eta_{2}\right)= & \alpha\left(\frac{1}{30} \eta_{1} \partial^{3} \eta_{2}-\frac{1}{30} \partial^{3} \eta_{1} \eta_{2}+\frac{1}{20} \partial^{2} \eta_{1} \partial \eta_{2}-\frac{1}{20} \partial \eta_{1} \partial^{2} \eta_{2}\right) \\
& -\frac{3 \hbar \beta}{10}\left(\frac{1}{2} \eta_{1} \partial^{3} \eta_{2}-\frac{1}{2} \partial^{3} \eta_{1} \eta_{2}-\frac{1}{2} \partial^{2} \eta_{1} \partial \eta_{2}+\frac{1}{2} \partial \eta_{1} \partial^{2} \eta_{2}\right), \\
L_{3}^{\varepsilon}\left(\eta_{1}, \eta_{2}, T\right)= & \beta\left(\eta_{1} \partial \eta_{2}-\partial \eta_{1} \eta_{2}\right) T .
\end{aligned}
$$

Please note the explicit first order quantum correction in $L_{2}^{\varepsilon}\left(\eta_{1}, \eta_{2}\right)$ and the infinitely many quantum corrections hidden in the $\hbar$ dependence of $\beta$.

\subsection{Quantum $\mathrm{L}_{\infty}$ relations with two symmetry parameters}

Having determined the quantum corrected $L_{n}$ products for the $\mathcal{W}_{3}$ algebra, let us now state and check the quantum $\mathrm{L}_{\infty}$ relations

$$
\mathcal{J}_{m+n}^{\mathrm{qu}}\left(\epsilon_{1}, \ldots, \epsilon_{m}, x_{1}, \ldots, x_{n}\right)+\sum_{\substack{\left(y_{1}, \ldots, y_{k}\right) \\ \rightarrow\left(x_{1}, \ldots, x_{n}\right)}} \hbar^{\xi} \mathcal{J}_{m+k}^{\mathrm{qu}}(\epsilon_{1}, \ldots, \epsilon_{m}, \underbrace{y_{1}, \ldots, y_{k}}_{\rightarrow\left(x_{1}, \ldots, x_{n}\right)})=0
$$

when plugging in exactly two symmetry parameters. These are the ones that are equivalent to the quantum closure condition (4.9).

\section{Quantum corrections to the $\mathbf{L}_{\infty}$ relations}

The distinguished new feature of the definition of quantum $\mathrm{L}_{\infty}$ algebras is the second term in (4.16) where the contractions appear. Let us therefore first list the $\mathrm{L}_{\infty}$ relations that are non-trivially corrected by such contraction terms.

Since we plug in two symmetry parameters and we need at least two fields to be able to contract, we must have at least four inputs in (4.16). But since the highest $L_{n}$ product is $L_{3}$, all relations $\mathcal{J}_{6}^{\mathrm{qu}}, \mathcal{J}_{7}^{\mathrm{qu}}, \cdots=0$ are automatically satisfied. To further trivialize most cases we can use that the only non-trivial $L_{3}$ products are $L_{3}^{W}(\eta, T, T)$ and $L_{3}^{W}\left(\eta_{1}, \eta_{2}, T\right)$. 
Since the first $L_{3}$ always maps into the kernel of the second $L_{3}$, for $\mathcal{J}_{5}^{\text {qu }} \sim L_{3} L_{3}$ one can conclude

$$
\mathcal{J}_{5}^{\mathrm{qu}}\left(\epsilon_{i}, \epsilon_{j}, \mathbf{W}, \mathbf{W}, \mathbf{W}\right)=0
$$

In a similar vein, evaluating (2.8) one finds that trivially

$$
\begin{aligned}
\mathcal{J}_{4}^{\text {qu }}(\varepsilon, \varepsilon, W, W) & =0, \\
\mathcal{J}_{4}^{\text {qu }}\left(\varepsilon_{1}, \varepsilon_{2}, \mathbf{W}, \mathbf{W}\right) & =0, \\
\mathcal{J}_{4}^{\text {qu }}(\varepsilon, \eta, W, T) & =0 .
\end{aligned}
$$

The only non-zero contraction terms can therefore arise in the terms

$$
\mathcal{J}_{4}^{\mathrm{qu}}(\epsilon, \eta, \overparen{T, T}), \quad \mathcal{J}_{4}^{\mathrm{qu}}\left(\eta_{1}, \eta_{2}, \overleftrightarrow{W, T}\right), \quad \mathcal{J}_{4}^{\mathrm{qu}}\left(\eta_{1}, \eta_{2}, \overparen{T, T}\right)
$$

From the form of the OPEs (4.1), one realizes that the contraction $T T$ yields terms proportional to $\hbar T$ and the identity $\hbar \mathbf{1}$, while the second contraction reads $W T \sim \hbar W$. Hence the $\mathrm{L}_{\infty}$ relations that are non-trivially corrected by a contraction of a higher $\mathrm{L}_{\infty}$ relation are

$$
\begin{aligned}
& 0=\mathcal{J}_{2}^{\mathrm{qu}}(\varepsilon, \eta)+\hbar \mathcal{J}_{4}^{\mathrm{qu}}(\varepsilon, \eta, \underbrace{T, T}_{\rightarrow 1}), \\
& 0=\mathcal{J}_{3}^{\mathrm{qu}}(\varepsilon, \eta, T)+\hbar \mathcal{J}_{4}^{\mathrm{qu}}(\varepsilon, \eta, \underbrace{T, T}_{\rightarrow T}), \\
& 0=\mathcal{J}_{2}^{\mathrm{qu}}\left(\eta_{1}, \eta_{2}\right)+\hbar \mathcal{J}_{4}^{\mathrm{qu}}(\eta_{1}, \eta_{2}, \underbrace{T, T}_{\rightarrow 1}), \\
& 0=\mathcal{J}_{3}^{\mathrm{qu}}\left(\eta_{1}, \eta_{2}, T\right)+\hbar \mathcal{J}_{4}^{\mathrm{qu}}(\eta_{1}, \eta_{2}, \underbrace{T, T}_{\rightarrow T}), \\
& 0=\mathcal{J}_{3}^{\mathrm{qu}}\left(\eta_{1}, \eta_{2}, W\right)+\hbar \mathcal{J}_{4}^{\mathrm{qu}}(\eta_{1}, \eta_{2}, \underbrace{T, W}_{\rightarrow W}) .
\end{aligned}
$$

Following the logic of section 3.1, we will now explicitly evaluate the contractions appearing in these quantum $\mathrm{L}_{\infty}$ relations. We start with terms arising from contractions of the $\mathrm{L}_{\infty}$ relation $\mathcal{J}_{4}^{\text {qu }}\left(\eta_{1}, \eta_{2}, T, T\right)$. In a first step we find

$$
\begin{aligned}
\mathcal{J}_{4}^{\mathrm{qu}}\left(\eta_{1}, \eta_{2}, T, T\right)= & -L_{2}^{T}\left(L_{3}^{\varepsilon}\left(\eta_{1}, \eta_{2}, T\right), T\right) \\
& +\frac{1}{2} L_{2}^{T}\left(\eta_{2}, L_{3}^{W}\left(\eta_{1}, T, T\right)\right)-\frac{1}{2} L_{2}^{T}\left(\eta_{1}, L_{3}^{W}\left(\eta_{2}, T, T\right)\right) .
\end{aligned}
$$

Recall that every $\mathrm{L}_{\infty}$ relation collects the contribution of the form $\left(\eta_{1} \eta_{2}\right)(T \star T)$. While the terms in the second line are already of this form, the first term is not, so that the non-associativity of the $\star$-product (3.2) is expected to induce contractions. Inserting the explicit expression of the $L_{n}$ products into the first term yields

$$
-L_{2}^{T}\left(L_{3}^{\varepsilon}\left(\eta_{1}, \eta_{2}, T\right), T\right)=-2 \beta(\partial(f T) \star T)-\beta((f T) \star \partial T),
$$


where we abbreviated $f:=\eta_{1} \partial \eta_{2}-\partial \eta_{1} \eta_{2}$. Using the normal ordering prescription (4.5) and its function linearity in the second argument we find for the first term

$$
\begin{gathered}
-2 \beta(\partial(f T) \star T)(z) \\
=-\beta\left(\oint \frac{d y}{2 \pi i} \frac{f(y) T(y) \circ T(z)}{(y-z)^{2}}+\partial f(z) N(T T)(z)+f(z) N(T \partial T)\right) \\
=-\beta\left(\frac{c \hbar}{240} \partial^{5} f(z)+\frac{\hbar}{3} \partial^{3} f(z) T(z)+\frac{\hbar}{2} \partial^{2} f(z) \partial T(z)\right. \\
+2 \partial f(z) N(T T)(z)+f(z) \partial N(T T)(z)) .
\end{gathered}
$$

Evaluating the second term in (4.22) similarly gives

$$
\begin{aligned}
& -\beta((f T) \star \partial T)(z)= \\
& \quad-\frac{\beta}{2}\left(\frac{c \hbar}{60} \partial^{5} f(z)+\frac{2 \hbar}{3} \partial^{3} f(z) T(z)+\frac{3 \hbar}{2} \partial^{2} f(z) \partial T(z)+f(z) \partial N(T T)\right) .
\end{aligned}
$$

Putting both terms together results in

$$
\begin{aligned}
-L_{2}^{T}\left(L_{3}^{\varepsilon}\left(\eta_{1}, \eta_{2}, T\right), T\right)= & -\frac{\beta \hbar c}{80} \partial^{5} f(z) \\
& -\frac{\beta \hbar}{3} \partial^{3} f(z) T(z)-\frac{5 \beta \hbar}{4} \partial^{2} f(z) \partial T(z)-\frac{\beta \hbar}{2} \partial f(z) \partial^{2} T(z) \\
& -2 \beta \partial f(z) N(T T)(z)-\frac{3 \beta}{2} f(z) \partial N(T T)(z)
\end{aligned}
$$

so that we can directly read off

$$
\begin{aligned}
& \hbar \mathcal{J}_{4}^{\mathrm{qu}}(\eta_{1}, \eta_{2}, \underbrace{T, T}_{\rightarrow \mathbf{1}})=-\frac{\beta \hbar c}{80} \partial^{5} f(z), \\
& \hbar \mathcal{J}_{4}^{\mathrm{qu}}(\eta_{1}, \eta_{2}, \underbrace{T, T}_{\rightarrow T})=-\frac{\beta \hbar}{3} \partial^{3} f(z) T(z)-\frac{5 \beta \hbar}{4} \partial^{2} f(z) \partial T(z)-\frac{\beta \hbar}{2} \partial f(z) \partial^{2} T(z) .
\end{aligned}
$$

Computing the other contractions is more lengthy, but follows the same steps. Let us therefore only state the results

$$
\begin{aligned}
\hbar \mathcal{J}_{4}^{\mathrm{qu}}(\varepsilon, \eta, \underbrace{T, T}_{\rightarrow T})= & -\frac{4 \hbar \beta}{3}\left(\partial \eta \partial^{3} \varepsilon-\frac{1}{2} \eta \partial^{4} \varepsilon\right) T-2 \hbar \beta\left(\partial \eta \partial^{2} \varepsilon-\frac{1}{3} \eta \partial^{3} \varepsilon\right) \partial T \\
& -\hbar \beta \eta \partial^{2} \varepsilon \partial^{2} T \\
\hbar \mathcal{J}_{4}^{\mathrm{qu}}(\varepsilon, \eta, \underbrace{T, T}_{\rightarrow \mathbf{1}})= & -\frac{\beta \hbar c}{40}\left(\partial \eta \partial^{5} \varepsilon+\frac{1}{2} \eta \partial^{6} \varepsilon\right),
\end{aligned}
$$

and finally

$$
\begin{aligned}
\hbar \mathcal{J}_{4}(\eta_{1}, \eta_{2}, \underbrace{T, W}_{\rightarrow W})= & -\frac{3 \beta \hbar}{4}\left(\partial \eta_{1} \partial^{2} \eta_{2}-\partial \eta_{2} \partial^{2} \eta_{1}\right) \partial W \\
& -\frac{3 \beta \hbar}{2} \partial^{2} f \partial W-\frac{9 \beta \hbar}{4} \partial f \partial^{2} W .
\end{aligned}
$$




\section{Checking the quantum $\mathbf{L}_{\infty}$ relations}

We are now in the position to state and check the quantum $\mathrm{L}_{\infty}$ relation with two symmetry parameters. We will sort them according to their appearance in the quantum closure condition (4.9) with $i, j, k \in\{T, W\}$.

- $(\mathbf{T T}, \mathbf{T})$ : the closure condition $(4.9)$ with $(i j, k)=(T T, T)$ is equivalent to

$$
\begin{aligned}
0 & =\mathcal{J}_{2}^{\mathrm{qu}}\left(\varepsilon_{1}, \varepsilon_{2}\right) \\
& =-L_{1}^{T}\left(L_{2}^{\varepsilon}\left(\varepsilon_{1}, \varepsilon_{2}\right)\right)+L_{2}^{T}\left(L_{1}^{T}\left(\varepsilon_{1}\right), \varepsilon_{2}\right)+L_{2}^{T}\left(\varepsilon_{1}, L_{1}^{T}\left(\varepsilon_{2}\right)\right)
\end{aligned}
$$

and

$$
\begin{aligned}
0 & =\mathcal{J}_{3}^{\mathrm{qu}}\left(\varepsilon_{1}, \varepsilon_{2}, T\right) \\
& =L_{2}^{T}\left(L_{2}^{\varepsilon}\left(\varepsilon_{1}, \varepsilon_{2}\right), T\right)+L_{2}^{T}\left(L_{2}^{T}\left(\varepsilon_{2}, T\right), \varepsilon_{1}\right)+L_{2}^{T}\left(L_{2}^{T}\left(T, \varepsilon_{1}\right), \varepsilon_{2}\right) .
\end{aligned}
$$

Inserting (4.13) these relations are readily checked to be satisfied.

- $(\mathbf{T T}, \mathbf{W})$ : there is only one non-trivial relation

$$
\begin{aligned}
0 & =\mathcal{J}_{3}^{\mathrm{qu}}\left(\varepsilon_{1}, \varepsilon_{2}, W\right) \\
& =L_{2}^{W}\left(L_{2}^{\varepsilon}\left(\varepsilon_{1}, \varepsilon_{2}\right), W\right)+L_{2}^{W}\left(L_{2}^{W}\left(\varepsilon_{2}, W\right), \varepsilon_{1}\right)+L_{2}^{W}\left(L_{2}^{W}\left(W, \varepsilon_{1}\right), \varepsilon_{2}\right),
\end{aligned}
$$

that is also directly satisfied.

- $(\mathbf{T W}, \mathbf{T})$ : one finds the single non-trivial relation

$$
\begin{aligned}
0 & =\mathcal{J}_{3}^{\mathrm{qu}}(\varepsilon, \eta, W) \\
& =L_{2}^{T}\left(L_{2}^{\eta}(\varepsilon, \eta), W\right)+L_{2}^{T}\left(L_{2}^{T}(\eta, W), \varepsilon\right)+L_{2}^{T}\left(L_{2}^{W}(W, \varepsilon), \eta\right) .
\end{aligned}
$$

As before, a short computation shows that this equation is satisfied without any constraints.

- $(\mathbf{T W}, \mathbf{W})$ : this is the first truly interesting case, as the closure condition involves a contribution from a contraction

$$
\begin{aligned}
& 0=\mathcal{J}_{2}^{\mathrm{qu}}(\varepsilon, \eta)+\hbar \mathcal{J}_{4}^{\mathrm{qu}}(\varepsilon, \eta, \underbrace{T, T}_{\rightarrow \mathbf{1}}), \\
& 0=\mathcal{J}_{3}^{\mathrm{qu}}(\varepsilon, \eta, T)+\hbar \mathcal{J}_{4}^{\mathrm{qu}}(\varepsilon, \eta, \underbrace{T, T}_{\rightarrow T}), \\
& 0=\mathcal{J}_{4}^{\mathrm{qu}}(\varepsilon, \eta, T, T) .
\end{aligned}
$$

When evaluating these relations, the contraction terms computed in (4.27) are crucial. Like in the classical case, the first equation is satisfied for $\alpha=2$. Note that terms from the quantum part of $L_{2}(\eta, T)$ get exactly canceled by the quantum correction from the contraction. The second equation is indeed satisfied for $\beta=\frac{16 \alpha}{5 c+22 \hbar}$, the value of the quantum $\mathcal{W}_{3}$ algebra. The third relation holds without giving any constraints on $\alpha, \beta$. 
- $(\mathbf{W W}, \mathbf{T})$ : in this case the closure is equivalent to the quantum $\mathrm{L}_{\infty}$ relations

$$
\begin{aligned}
& 0=\mathcal{J}_{2}^{\mathrm{qu}}\left(\eta_{1}, \eta_{2}\right)+\hbar \mathcal{J}_{4}^{\mathrm{qu}}(\eta_{1}, \eta_{2}, \underbrace{T, T}_{\rightarrow \mathbf{1}}), \\
& 0=\mathcal{J}_{3}^{\mathrm{qu}}\left(\eta_{1}, \eta_{2}, T\right)+\hbar \mathcal{J}_{4}^{\mathrm{qu}}(\eta_{1}, \eta_{2}, \underbrace{T, T}_{\rightarrow T}), \\
& 0=\mathcal{J}_{4}^{\mathrm{qu}}\left(\eta_{1}, \eta_{2}, T, T\right) .
\end{aligned}
$$

Again, the contraction terms (4.26) are needed. The first equation is satisfied for $\alpha=2$ and the second for $\beta=\frac{16 \alpha}{5 c+22 \hbar}$. Again, the quantum corrected $\mathrm{L}_{\infty}$ relations fix the open constants exactly to the values expected for the quantum $\mathcal{W}_{3}$ algebra. The third equation holds independently of the numerical values of $\alpha, \beta$.

- $(\mathbf{W W}, \mathbf{W})$ : the quantum $\mathrm{L}_{\infty}$ relations equivalent to closure are

$$
\begin{aligned}
& 0=\mathcal{J}_{3}^{\mathrm{qu}}\left(\eta_{1}, \eta_{2}, W\right)+\hbar \mathcal{J}_{4}^{\mathrm{qu}}(\eta_{1}, \eta_{2}, \underbrace{T, W}_{\rightarrow W}), \\
& 0=\mathcal{J}_{4}^{\mathrm{qu}}\left(\eta_{1}, \eta_{2}, T, W\right) .
\end{aligned}
$$

After inserting the contraction term (4.28), both equations hold independent of $\alpha$ and $\beta$.

\section{5 $\mathrm{L}_{\infty}$ relations with three symmetry parameters}

After we have checked the $\mathrm{L}_{\infty}$ relations with two symmetry parameters, it remains to evaluate those with three symmetry parameters. Recall that these are equivalent to the Jacobi identity

$$
\sum_{\text {cycl }}\left[\delta_{\varepsilon_{i}}^{\mathrm{qu}},\left[\delta_{\varepsilon_{j}}^{\mathrm{qu}}, \delta_{\varepsilon_{k}}^{\mathrm{qu}}\right]\right]=0
$$

For three symmetry parameter insertions, $\mathcal{J}_{n}=0$ is trivially satisfied for $n \geq 5$ in the case of the $\mathcal{W}_{3}$ algebra. Therefore, there cannot be any correction terms arising from contractions. Again sorting them according to the triplet $(i j k)$ in $(4.36)$, the quantum $\mathrm{L}_{\infty}$ relations read as follows:

- (TTT):

$$
0=L_{2}^{\varepsilon}\left(L_{2}^{\varepsilon}\left(\varepsilon_{1}, \varepsilon_{2}\right), \varepsilon_{3}\right)+L_{2}^{\varepsilon}\left(L_{2}^{\varepsilon}\left(\varepsilon_{3}, \varepsilon_{1}\right), \varepsilon_{2}\right)+L_{2}^{\varepsilon}\left(L_{2}^{\varepsilon}\left(\varepsilon_{2}, \varepsilon_{3}\right), \varepsilon_{1}\right) .
$$

- (TTW):

$$
0=L_{2}^{\eta}\left(L_{2}^{\varepsilon}\left(\varepsilon_{1}, \varepsilon_{2}\right), \eta\right)+L_{2}^{\eta}\left(L_{2}^{\eta}\left(\eta, \varepsilon_{1}\right), \varepsilon_{2}\right)+L_{2}^{\eta}\left(L_{2}^{\eta}\left(\varepsilon_{2}, \eta\right), \varepsilon_{1}\right) .
$$

- (WWT):

$$
\begin{aligned}
0= & L_{2}^{\varepsilon}\left(L_{2}^{\varepsilon}\left(\eta_{1}, \eta_{2}\right), \varepsilon\right)+L_{2}^{\varepsilon}\left(L_{2}^{\eta}\left(\varepsilon, \eta_{1}\right), \eta_{2}\right)+L_{2}^{\varepsilon}\left(L_{2}^{\eta}\left(\eta_{2}, \varepsilon\right), \eta_{1}\right) \\
& +L_{3}^{\varepsilon}\left(\eta_{1}, \eta_{2}, L_{1}^{T}(\varepsilon)\right) \\
0= & -L_{2}^{\varepsilon}\left(L_{3}^{\varepsilon}\left(\eta_{1}, \eta_{2}, T\right), \varepsilon\right)+L_{3}^{\varepsilon}\left(L_{2}^{\eta}\left(\eta_{1}, \varepsilon\right), \eta_{2}, T\right) \\
& -L_{3}^{\varepsilon}\left(L_{2}^{\eta}\left(\eta_{2}, \varepsilon\right), \eta_{1}, T\right)+L_{3}^{\varepsilon}\left(L_{2}^{T}(T, \varepsilon), \eta_{1}, \eta_{2}\right) .
\end{aligned}
$$


The first $\mathcal{J}_{3}$-type relation requires $\beta=\frac{16 \alpha}{5 c+22 \hbar}$ to hold and, due to the appearance of the non-vanishing last term, features that the two-product $L_{2}$ violates its Jacobi identity.

- (WWW):

$$
\begin{aligned}
0= & L_{2}^{\eta}\left(L_{2}^{\varepsilon}\left(\eta_{1}, \eta_{2}\right), \eta_{3}\right)+L_{2}^{\eta}\left(L_{2}^{\varepsilon}\left(\eta_{3}, \eta_{1}\right), \eta_{2}\right)+L_{2}^{\eta}\left(L_{2}^{\varepsilon}\left(\eta_{2}, \eta_{3}\right), \eta_{1}\right), \\
0= & L_{2}^{\eta}\left(L_{3}^{\varepsilon}\left(\eta_{1}, \eta_{2}, T\right), \eta_{3}\right)+L_{2}^{\eta}\left(L_{3}^{\varepsilon}\left(\eta_{3}, \eta_{1}, T\right), \eta_{2}\right)+L_{2}^{\eta}\left(L_{3}^{\varepsilon}\left(\eta_{2}, \eta_{3}, T\right), \eta_{1}\right), \\
0= & L_{3}^{\varepsilon}\left(L_{2}^{T}\left(\eta_{1}, W\right), \eta_{2}, \eta_{3}\right)+L_{3}^{\varepsilon}\left(L_{2}^{T}\left(\eta_{2}, W\right), \eta_{3}, \eta_{1}\right) \\
& +L_{3}^{\varepsilon}\left(L_{2}^{T}\left(\eta_{3}, W\right), \eta_{1}, \eta_{2}\right) .
\end{aligned}
$$

\section{$5 \quad$ Summary and conclusions}

This completes the proof that the quantum $\mathcal{W}_{3}$ algebra is an example for a quantum $\mathrm{L}_{\infty}$ algebra as defined in section 3.1. Like for the classical $\mathcal{W}_{3}$ algebra, the quantum corrected relations with two inputs gave the constraint $\alpha=2$ and the relations with three inputs $\mathcal{J}_{3}^{\text {qu }}=0$ required $\beta=\frac{16 \alpha}{5 c+22 \hbar}$. The only other non-trivial higher order relations were satisfied without any further constraint. The $\mathrm{L}_{\infty}$ relations with three symmetry parameters were essentially the same as in the classical case.

Let us emphasize that the quantum contractions in (3.19) are necessary for the $\mathrm{L}_{\infty}$ relations to hold. This means that the quantum $\mathcal{W}_{3}$ algebra does neither define a classical nor a loop $\mathrm{L}_{\infty}$ algebra (as appeared for CSFT), but this new type of a quantum $\mathrm{L}_{\infty}$ algebra. Of course the higher products in CSFT and for quantum $\mathcal{W}$ algebras are different from the onset. In the latter case they involve the non-associative normal ordered product of 2d CFT, whereas in the former case they are the loop corrected n-vertices of CSFT. Thus, it seems that for global and gauge symmetries there does not exist a unique version of a physically reasonable definition of an $\mathrm{L}_{\infty}$ algebra for a quantum theory.

We expect that in general the whole class of $\mathcal{W}$ algebras yields further examples for quantum $\mathrm{L}_{\infty}$ algebras, since all of them have a closing symmetry algebra that involves normal ordered products as defined in CFT. As in the classical case, also higher $n$-products will be non-trivial. Since our analysis of quantum W-algebras is restricted to non-trivial elements in $X_{0} \oplus X_{-1}$, it is not obvious whether and how this structure generalizes to more general gradings.

\section{Acknowledgments}

We are grateful to Andreas Deser for discussions.

Open Access. This article is distributed under the terms of the Creative Commons Attribution License (CC-BY 4.0), which permits any use, distribution and reproduction in any medium, provided the original author(s) and source are credited. 


\section{References}

[1] B. Zwiebach, Closed string field theory: Quantum action and the B-V master equation, Nucl. Phys. B 390 (1993) 33 [hep-th/9206084] [INSPIRE].

[2] F.A. Berends, G.J.H. Burgers and H. van Dam, On the Theoretical Problems in Constructing Interactions Involving Higher Spin Massless Particles, Nucl. Phys. B 260 (1985) 295 [INSPIRE].

[3] T. Lada and J. Stasheff, Introduction to SH Lie algebras for physicists, Int. J. Theor. Phys. 32 (1993) 1087 [hep-th/9209099] [InSPIRE].

[4] D. Roytenberg and A. WEinstein, Courant Algebroids and Strongly Homotopy Lie Algebras, math/9802118 [INSPIRE].

[5] R. Fulp, T. Lada and J. Stasheff, sh-Lie algebras induced by gauge transformations, Commun. Math. Phys. 231 (2002) 25 [inSPIRE].

[6] A. Deser and C. Sämann, Extended Riemannian Geometry I: Local Double Field Theory, arXiv: 1611.02772 [INSPIRE].

[7] A. Sen, Wilsonian Effective Action of Superstring Theory, JHEP 01 (2017) 108 [arXiv: 1609.00459] [INSPIRE].

[8] O. Hohm and B. Zwiebach, $L_{\infty}$ Algebras and Field Theory, Fortsch. Phys. 65 (2017) 1700014 [arXiv:1701.08824] [INSPIRE].

[9] R. Blumenhagen, M. Fuchs and M. Traube, $\mathcal{W}$ algebras are $L_{\infty}$ algebras, JHEP 07 (2017) 060 [arXiv: 1705.00736] [INSPIRE].

[10] P. Bouwknegt and K. Schoutens, W symmetry in conformal field theory, Phys. Rept. 223 (1993) 183 [hep-th/9210010] [INSPIRE].

[11] M. Markl, Loop homotopy algebras in closed string field theory, Commun. Math. Phys. 221 (2001) 367 [hep-th/9711045] [INSPIRE].

[12] G. Burgers, On the construction of field theories for higher spin massless particles, Ph.D. Thesis, Rijksuniversiteit te Leiden (1985).

[13] P. Di Francesco, P. Mathieu and D. Sénéchal, Conformal Field Theory, Graduate Texts in Contemporary Physics, Springer (1997).

[14] A.B. Zamolodchikov, Infinite Additional Symmetries in Two-Dimensional Conformal Quantum Field Theory, Theor. Math. Phys. 65 (1985) 1205 [InSPIRE].

[15] R. Blumenhagen, M. Flohr, A. Kliem, W. Nahm, A. Recknagel and R. Varnhagen, W algebras with two and three generators, Nucl. Phys. B 361 (1991) 255 [InSPIRE].

[16] R. Blumenhagen and E. Plauschinn, Introduction to conformal field theory, Lect. Notes Phys. 779 (2009) 1 [INSPIRE].

[17] P. Bowcock and G.M.T. Watts, On the classification of quantum $W$ algebras, Nucl. Phys. B 379 (1992) 63 [hep-th/9111062] [INSPIRE].

[18] V. Kac, Vertex algebras for beginners, American Mathematical Society (1996). 